\title{
Adult onset supranuclear ophthalmoplegia, cerebellar ataxia, and neurogenic proximal muscle weakness in a brother and sister: another hexosaminidase A deficiency syndrome
}

\author{
A E HARDING,* E P YOUNG, $†$ F SCHON* \\ From the National Hospitals for Nervous Diseases and Institute of Neurology, ${ }^{*}$ and the Institute of Child \\ Health, $\dagger$ London, $U K$
}

SUMMARY An Ashkenazi Jewish brother and sister developed progressive ataxia and proximal neurogenic muscle weakness, associated with supranuclear ophthalmoplegia, in the fourth decade of life. Hexosaminidase A activity, assayed using both synthetic and natural substrates, was severely reduced in the patients' plasma, leukocytes, and skin fibroblasts. Enzyme activity in their parents was in a similar range to that seen in heterozygotes for Tay-Sachs disease. The increasing evidence for marked clinical and molecular heterogeneity in the $\mathrm{GM}_{2}$ gangliosidoses warrants their consideration in the diagnosis of multisystem degenerative neurological disorders, even if onset of symptoms is in adult life.

The $\mathrm{GM}_{2}$ gangliosidoses result from genetically determined deficiencies of the enzyme hexosaminidase which cleaves the terminal $\mathrm{N}$ acetylgalactosamine moiety from $\mathrm{GM}_{2}$ ganglioside. Hexosaminidase A deficiency was first recognised in patients with Tay-Sachs disease, a progressive infantile encephalopathy comprising progressive dementia associated with seizures and the macular cherry red spot. ${ }^{1}$ In the last decade it has become apparent that the hexosaminidase deficiencies exhibit marked clinical and genetic heterogeneity. Neurological symptoms may develop in infancy, childhood, or adult life, with rapid or slow progression. Enormous phenotypic variation has been observed in deficient patients; the clinical features reported to date have included dementia, psychosis, seizures, ataxia, dystonia, spasticity, and neurogenic muscle weakness, either alone or in various combinations. ${ }^{2}$

This paper describes a brother and sister with hexosaminidase A deficiency and an unusual clinical syndrome of progressive supranuclear ophthalmoplegia, cerebellar ataxia, and neurogenic proximal muscle weakness. Neurological symptoms did not develop until the fourth decade of life.

Address for reprint requests: Dr A E Harding, Department of Clinical Neurology, Institute of Neurology, Queen Square, London WCIN 3BG, UK.

Received 3 June 1986. Accepted 15 August 1986

\section{Case reports}

Case 1 This 50 year old female developed normally in infancy and childhood but was never athletic, finding it difficult to ride a bicycle. She was unaware of any definite neurological symptoms until the age of 35 years, when she first noticed progressive unsteadiness on walking and became unable to rise from a low chair or climb stairs without using her arms. She can now only walk with assistance due to a combination of ataxia and muscle weakness.

On examination, she spoke rapidly with a mixed cerebellar/bulbar dysarthria, and had a staring gaze. The fundi were normal. Voluntary upgaze was limited to $10 \%$ of normal, and horizontal and downgaze to about $80 \%$. Pursuit improved the range of eye movements, to about $30 \%$ of normal on upgaze, but with some impersistence. The doll's head manoeuvre resulted in full horizontal movements and downgaze, and a further improvement in upgaze. Convergence was poor. Occasional facial twitching was seen, particularly around the left side of the mouth. There were brisk jaw, pout, and gag reflexes. Voluntary palatal and tongue movements were reduced. There was moderate wasting of the thighs with marked (MRC grade 3) weakness of all movements of the hips and knees. The knee jerks were absent but tapping the patellar tendons produced a brisk adductor response bilaterally; the other tendon reflexes were normal and the plantar responses were extensor. There was mild limb ataxia. Vibration sense was reduced below the knees but other sensory modalities were normal. The patient's gait was ataxic and waddling; she could not stand from sitting without using her arms. 

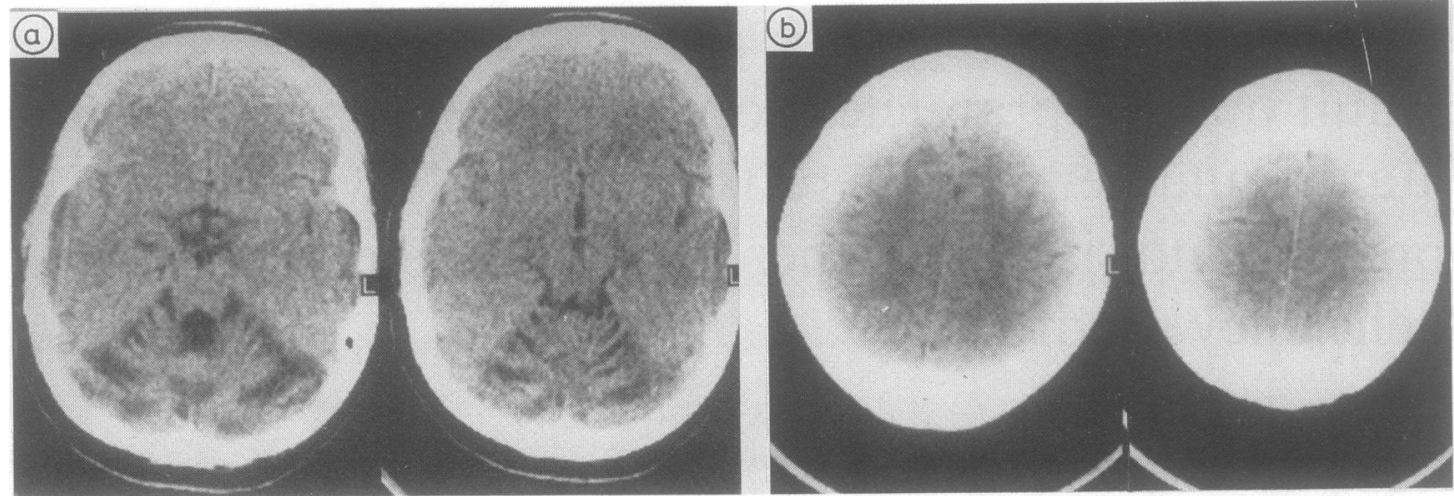

Fig Case 1 (a) CT scan showing gross cerebellar and brainstem atrophy; (b) the cerebral hemispheres have a normal appearance.

Case 2 The 45 year old brother of Case 1 was perhaps always slightly clumsy, but completed military service and was graded Al in medical examinations. He was able to run about 12 miles $(20 \mathrm{~km})$ in his early twenties. Around the age of 30 years he became aware of progressive clumsiness of his limbs, more on the right than the left, and unsteadiness of gait. His disability has become more noticeable in the last five years, with frequent falls, and he has developed symptoms suggesting proximal weakness of his legs.

On examination, the findings were similar to those seen in Case 1. The eye movement disorder and muscle weakness were slightly less prominent but he had more marked ataxia in the limbs. The tendon reflexes and sensation were normal.

The patients were members of an Ashkenazi Jewish family which originated from eastern Europe. They had no other sibs. Their unrelated parents were clinically normal at the ages of 77 and 71 .

Investigations in both cases showed normal routine haematological and biochemical profiles. The following were also normal or negative: serum lactate dehydrogenase, leucocyte glutamate dehydrogenase activity, syphilis serology, serum vitamin B12, visual and somatosensory evoked potentials, and motor and sensory nerve conduction studies.
The serum creatine kinase was $50 \mathrm{iu} / 1$ in Case 1 and $97 \mathrm{iu} / 1$ in Case 2 (normal 10-70). Electromyography showed evidence of denervation in proximal and distal muscles in both patients, and this was confirmed by muscle biopsy in Case 1 . Electronystagmography in Case 1 showed hypometric pursuit and horizontal saccades but saccadic velocities were normal. Optokinetic nystagmus was abnormal with ocular deviation in the direction of the slow component. Occasional square wave jerks were seen. CT brain scans showed gross cerebellar and brainstem atrophy, but the cerebral hemispheres appeared normal (fig). Psychometric testing suggested mild intellectual deterioration with some perceptual impairment but both patients scored 111 for verbal IQ on the WAIS scale.

Table 1 shows the hexosaminidase activity found in plasma and leukocytes from both patients and their parents, and also in the patients' skin fibroblasts, using the synthetic 4-methyl-umbelliferyl substrate as described previously. ${ }^{3}$ Hexosaminidase A activity was markedly reduced in the patients, to the same extent as seen in cases of Tay-Sachs disease; in their parents activity was in the range seen in Tay-Sachs heterozygotes. Table 2 shows $\mathbf{G M}_{2}$ degradation in cultured skin fibroblasts from both patients, measured in

Table 1 Hexosaminidase activity in Cases 1 and 2 and their parents

\begin{tabular}{|c|c|c|c|c|c|c|}
\hline & \multicolumn{3}{|c|}{ Total $\beta$ hexosaminidase activity ( $\mu \mathrm{mol} / \mathrm{mg}$ protein/h) } & \multicolumn{3}{|c|}{$\%$ hexosaminidase $A$} \\
\hline & Plasma & $W B C$ & Skin fibroblasts & Plasma & $\boldsymbol{W B C}$ & Skin fibroblasts \\
\hline $\begin{array}{l}\text { Case } 1 \\
\text { Case } 2 \\
\text { Mother } \\
\text { Father }\end{array}$ & $\begin{array}{l}0.5 \\
0.4 \\
1.3 \\
0.9\end{array}$ & $\begin{array}{l}1 \cdot 5 \\
1.6 \\
1.5 \\
1.7\end{array}$ & $\begin{array}{l}6 \cdot 5 \\
5 \cdot 2 \\
- \\
-\end{array}$ & $\begin{array}{l}14 \\
10 \\
39 \\
46\end{array}$ & $\begin{array}{r}5 \\
2 \\
38 \\
37\end{array}$ & $\begin{array}{r}6 \\
6 \\
- \\
-\end{array}$ \\
\hline $\begin{array}{l}\text { TSD* } \\
\mathrm{n}\end{array}$ & $\begin{array}{l}0 \cdot 3-1 \cdot 3 \\
9\end{array}$ & $\begin{array}{l}0 \cdot 7-1.9 \\
18\end{array}$ & $10^{2 \cdot 4-10 \cdot 3}$ & $\begin{array}{c}0-5 \\
10^{-5}\end{array}$ & 29 & $\begin{array}{l}2-13 \\
8\end{array}$ \\
\hline $\begin{array}{l}\text { Obligate TSD } \\
\text { heterozygotes* }\end{array}$ & $\begin{array}{l}0.5-1 \cdot 4 \\
14\end{array}$ & $\begin{array}{l}0 \cdot 7-2 \cdot 4 \\
25\end{array}$ & $\begin{array}{l}3 \cdot 7-7 \cdot 4 \\
3\end{array}$ & $\begin{array}{l}32-52 \\
16\end{array}$ & $\begin{array}{l}29-48 \\
40\end{array}$ & $\underset{3}{31-38}$ \\
\hline
\end{tabular}

*Data from Professor A D Patrick's laboratory, Institute of Child Health, London.

TSD $=$ Tay-Sachs disease. 
Table $2 G M_{2}$ degradation in cultured skin fibroblasts (pmol/hr/mg/activator unit)

\begin{tabular}{ll}
\hline Case 1 & $12 \cdot 5$ \\
Case 2 & $2 \cdot 57$ \\
Normal controls* & $510 \pm 150$ \\
Heterozygotes* & $330 \pm 140$ \\
GM Gangliosidosis:* $_{\text {Infantile (TSD) }}$ & $<5$ \\
Late infantile & $5-10$ \\
Juvenile and adult & $17 \pm 5$ \\
\hline
\end{tabular}

*Data from Professor K Sandhoff's laboratory, Bonn University.

Professor K Sandhoff's laboratory according to the method of Conzelmann and colleagues. ${ }^{4}$ The rates observed were in the Tay-Sachs disease range in Case 2, and rather higher, similar to those found in other cases of juvenile and adult onset $\mathrm{GM}_{2}$ gangliosidosis, in Case 1. Electron microscopy of samples of skin from both patients, and muscle from Case 1, showed no intraneuronal inclusions.

\section{Discussion}

Normal hexosaminidase activity requires two major isozymes (hexosaminidases A and B), three gene loci, two kinds of subunits (alpha and beta, encoded for on chromosomes 15 and 5 respectively), and an activator protein. Hexosaminidase $A$ is composed of both alpha and beta subunits, whereas hexosaminidase B consists only of beta chains. Mutations of the beta locus result in deficiencies of hexosaminidase $A$ and B; the commonest phenotype is Sandhoff disease, an infantile encephalopathy, although cases with juvenile and adult onset have been described. ${ }^{5}$ Deficiency of the activator protein gives rise to the "AB variant" of Tay-Sachs disease, but has also been described in a young adult with dementia, seizures, and communicating hydrocephalus. ${ }^{6}$

Classical infantile Tay-Sachs disease is caused by a mutation at the alpha locus ${ }^{2}$ which results in virtually undetectable activity of hexosaminidase A. ${ }^{1}$ Patients with late infantile or juvenile onset variants of TaySachs disease have also been described. ${ }^{3}$ In the latter, affected children develop normally until about the age of four years, when gait ataxia and progressive dementia become apparent, associated with seizures and spasticity, leading to death in the second decade of life.

More chronic neurological disorders associated with hexosaminidase A deficiency have been reported in about 20 patients, a number of whom were identified during screening programmes for TaySachs disease carriers amongst the Ashkenazi Jewish population. ${ }^{7-11}$ Most of the cases described had a variable combination of cerebellar ataxia and neurogenic proximal muscle weakness, mainly affecting the legs, with ages of onset of symptoms ranging from two to 25 years. Other features included facial grimacing, psychosis, dystonia, dementia and, rarely, peripheral nerve involvement. In some patients the overall clinical picture has resembled that of amyo- trophic lateral sclerosis. ${ }^{11}$

The cases reported here are unusual in their late age of onset of symptoms, although in retrospect they admitted to lifelong clumsiness. The latter has been noted in previous reports. ${ }^{910} \mathrm{~A}$ staring gaze and abnormal optokinetic nystagmus have been reported in hexosaminidase $\mathrm{A}$ deficiency $;^{78}$ both of our patients had a more marked disorder of eye movements which appeared to be supranuclear in origin. Their rapid dysarthric speech was unusual and this has also been described by other authors. ${ }^{79}$

The reason for the seemingly selective deleterious effect of ganglioside accumulation on the cerebellum, brainstem, and spinal cord is unclear, as is the case in most genetically determined neurodegenerative disorders. The CT scan appearances in our patients were particularly striking. Marked cerebellar atrophy, seen on magnetic resonance imaging, was also reported by Mitsumoto and colleagues ${ }^{11}$ in two patients who were only mildly ataxic. Necropsy of one adult hexosaminidase A deficient patient revealed predominant $\mathrm{GM}_{2}$ storage and neuronal loss in the cerebellum and subcortical structures rather than in the cerebral cortex; the reverse occurs in infantile Tay-Sachs disease. ${ }^{7}$

The patients reported here had levels of hexosaminidase $A$ activity similar to those seen in infantile Tay-Sachs disease when the enzyme was assayed using a synthetic substrate. Activity was higher with natural substrate in Case 1, about $12.5 \%$ of normal, as has been observed previously in deficient patients with later onset and less rapidly progressive neurological disability. ${ }^{12}$ The relative increase in hexosaminidase $B$, which accounts for the normal total hexosaminidase activity, is usual in hexosaminidase A deficiency.

It is possible that our patients are compound heterozygotes for the Tay-Sachs disease gene and another mutation at the alpha locus; the four sibs described by Navon and colleagues ${ }^{9}$ were very similar to them clinically, and two of these had offspring with Tay-Sachs disease. Johnson ${ }^{2}$ suggested that the clinical complexity of the hexosaminidase deficiencies could be best understood if a parellel were drawn between them and the haemoglobinopathies, of which there are nearly 400 . Multiple gene loci are involved in the synthesis of both haemoglobin and the hexosaminidase enzyme system. If the likely assumption is made that different mutations occur at the alpha and beta loci, and that at least some compound heterozygotes manifest neurological disease, it will be appreciated that the potential for clinical and genetic heterogeneity within hexosaminidase deficiency states is enormous. It is worth considering this diagnosis in patients with multisystem degenerative neurological disorders, even if onset is in adult life. Accurate diagnosis may have important implications for genetic counselling. 
We thank Dr P C Gautier-Smith for permission to report patients under his care, and Professor $\mathrm{K}$ Sandhoff for performing the $\mathbf{G M}_{2}$ degradation studies.

\section{References}

1 Okada S, O'Brien JS. Tay-Sachs disease: generalised absence of a beta-D-N-acetylhexosaminidase component. Science 1969;165:698-700.

2 Johnson WG. The clinical spectrum of hexosaminidase deficiency diseases. Neurology 1981;31:1453-6.

3 Brett EM, Ellis RB, Haas L, et al. Late onset $\mathrm{GM}_{2}$ gangliosidosis. Clinical, pathological and biochemical studies on eight patients. Arch Dis Child 1973; 48:775-85.

4 Conzelmann E, Kytzia H-J, Navon R, Sandhoff K. Ganglioside $\mathrm{GM}_{2} \quad \mathrm{~N}$-acetyl- $\beta$-D-galactosaminidase activity in cultured fibroblasts of late infantile and adult $\mathrm{GM}_{2}$ gangliosidosis patients and of healthy probands with low hexosaminidase levels. Am J Hum Genet 1983;35:900-13.

5 Oonk JGW, van der Helm HJ, Martin JJ. Spinocerebellar degeneration: hexosaminidase $\mathbf{A}$ and $\mathrm{B}$ deficiency in two adult sisters. Neurology 1979; 31:151-6.
6 O'Neill B, Butler AB, Young E, Falk PM, Bass NH. Adult onset $\mathrm{GM}_{2}$ gangliosidosis. Neurology 1978;28:1117-23.

7 Rapin I, Suzuki K, Suzuki K, Valsamis MP. Adult (chronic) $\mathrm{GM}_{2}$ gangliosidosis-atypical spinocerebellar degeneration in a Jewish sibship. Arch Neurol 1976;33:120-30.

8 Willner JP, Grabowski GA, Gordon RE, Bender AN, Desnick RJ. Chronic $\mathrm{GM}_{2}$ gangliosidosis masquerading as atypical Friedreich ataxia: clinical, morphologic and biochemical studies of nine cases. Neurology 1981;31:787-98.

9 Navon R, Argov Z, Brand N, Sandback U. Adult GM gangliosidosis in association with Tay-Sachs disease: a new phenotype. Neurology 1981;31:1397-401.

10 Johnson WG, Wigger HJ, Karp HR, Glaubiger LM, Rowland LP. Juvenile spinal muscular atrophy: a new hexosaminidase deficiency phenotype. Ann Neurol 1982;11:11-16.

11 Mitsumoto H, Sliman R, Schafer IA, et al. Motor neuron disease and adult hexosaminidase A deficiency in two families: evidence for multisystem degeneration. Ann Neurol 1985;17:378-85.

12 O'Brien JS. The gangliosidoses. In: Stanbury JB, Wyngaarden JB, Fredrickson DS, Goldstein JL, Brown MS, eds. The Metabolic Basis of Inherited Disease. 5th ed. New York: McGraw Hill, 1983:945-72. 